\title{
Arbeitsschutz
}

\section{So lassen sich Praxisunfälle vermeiden}

\author{
Nadelstichverletzungen sind die Klassiker, wenn es um Unfälle in der \\ Arztpraxis geht. Doch es gibt noch mehr Gefahrenquellen, gegen die \\ sich Praxischefs wappnen müssen.
}

$\mathrm{E}$ in rutschiger Boden, falsche Schuhe, Hektik - es sind meistens Banalitäten, die im Praxisalltag zu Stolperfallen und bösen Stürzen führen. „Speziell im Gesundheitswesen treten häufig Stichverletzungen durch kontaminierte Nadeln auf“, weiß Christopher Bulle, Projektleiter Qualitätsmanagement bei dem Beratungsunternehmen medicteach. „Das ist mir als Rettungsassistent im Notdienst schon selbst passiert“, erzählt Bulle. „Typisch ist auch, wenn Praxismitarbeiter mit Birkenstock-Schuhen oder Crocs im Winter mal eben über die Straße zur Apotheke laufen oder zum Rauchen gehen.“

Bulle schult Ärzte in Workshops der Kassenärztlichen Vereinigung Hessen über „Gefährdungsbeurteilung in Arztpraxen“. Aufgrund neuer gesetzlicher Vorschriften wird der Arbeitsschutz für die Praxisorganisation immer wichtiger. Bis Mai 2013 musste zum Beispiel die Biostoffverordnung auf Basis der EURichtlinie 2010/32/EU überarbeitet werden. Die Richtlinie sieht Regelungen vor, um Verletzungen durch scharfe oder spitze Instrumente im Gesundheitssektor $\mathrm{zu}$ vermeiden. Auch die geplanten Änderungen des Infektionsschutzgesetzes, mit denen anerkannte Empfehlungen zur Hygiene verbindlich für Ärzte werden sollen, setzen die Praxen unter Zugzwang.

Sicherheitsbeauftragter teils Pflicht Grundsätzlich ist die Zuständigkeit für den Arbeits- und Gesundheitsschutz der Praxismitarbeiter Chefsache. Wie die Berufsgenossenschaft für Gesundheitsdienst und Wohlfahrtspflege (BGW) bestätigt, ist jeder Arbeitgeber für den Arbeitsschutz derjenigen Mitarbeiter ver- antwortlich, mit denen er einen Arbeitsvertrag abgeschlossen hat. Beschäftigen Inhaber einer Praxisgemeinschaft Personal gemeinsam über eine Gesellschaft, kann einer der Gesellschafter als Verantwortlicher für den Arbeitsschutz benannt werden. Praxen mit mehr als 20 Mitarbeitern müssen einen Sicherheitsbeauftragten benennen, der dafür sorgt, den Arbeits- und Gesundheitsschutz zu verbessern. Bevor ein Plan mit konkreten Maßnahmen zum Arbeitsschutz erstellt wird, sollten zunächst in einer Art Brainstorming mit den Mitarbeitern alle möglichen Gefahren erfasst werden, denn sie wissen am besten, welche Gefährdungen und Belastungen an ihren jeweiligen Arbeitsplätzen auftreten können. „Nicht zu vergessen sind mögliche psychische Belastungen, etwa ein getrübtes Arbeitsklima oder schwierige Patienten“, so Bulle.

\section{Maßnahmenkontrolle notwendig}

Für Ärzte ist es nicht leicht, einzelne Gefahren richtig einzuschätzen. Zwar gibt es für einige Gefährdungen und Belastungen Sicherheitsnormen und Grenzwerte in Gesetzen, Unfallverhütungsvorschriften und Technische Regeln. Viele Gefahren lassen sich jedoch nicht in Normen fassen. Die BGW rät, auch mal etwas mehr als das gesetzlich Notwendige zu tun. Die Technische Regel schreibt bei Latexallergie etwa einen Proteingehalt von weniger als 30 Mikrogramm pro Gramm Handschuh vor normalerweise unproblematisch, doch für Mitarbeiter mit entzündeter Haut riskant.

Die Gefährdungsbeurteilung erstreckt sich auf bestimmte Räumlichkeiten, wie Labor, einzelne Abläufe, wie den Reini- gungsdienst, und auf bestimmte Personengruppen, wie Jugendliche, Behinderte, Schwangere und Allergiker. Alle ermittelten Gefahrenquellen sowie die daraus abgeleiteten und umgesetzten Maßnahmen müssen laut Arbeitsschutzgesetz schriftlich dokumentiert werden. Dazu gehört auch, nach einem halben Jahr zu kontrollieren, wie effektiv die einzelnen Maßnahmen waren. In Praxen mit weniger als zehn Mitarbeitern genügt übrigens eine vereinfachte Dokumentation. Grundlage sind hier die Vorschriften der Berufsgenossenschaften (BGV Anlage 1 und 3).

\section{Strafen sind selten}

Eine schriftliche Dokumentation hilft auch bei Prüfungen durch die Gesundheitsbehörden. Die Gewerbeaufsicht erscheint meist unangemeldet und kontrolliert etwa, ob die Praxen sichere Injektionssysteme verwenden. Die Gesundheitsämter prüfen, wie streng Hygienevorschriften eingehalten werden. „Wer dann eine aktuelle Gefährdungsbeurteilung aus dem Rechner zaubern kann, hinterlegt mit Unterweisungsnachweisen, Medizinprodukte-Unterlagen und einem Hygieneplan, ist schon mal auf der sicheren Seite", betont Bulle. "Gesundheitsämter fragen auch nach der Biostoffverordnung, die separat aufgelistet werden muss.“ Verstöße gegen Arbeitsschutz- oder Hygienevorschriften können Folgen haben. Zwar seien laut Bulle Strafen selten, doch „im Schadensfall werden Berufsgenossenschaft und im schlimmsten Fall auch Staatsanwaltschaft genau hinschauen, ob alle Bestimmungen befolgt wurden. Die Berufsgenossenschaft verlangt öfter Regresszahlungen."

Stefan Holler 